\title{
Fast geometrical characterization of vertical cracks using burst vibrothermography
}

\author{
by A. Mendioroz*, R. Celorrio**, A Cifuentes ${ }^{* * *}$ and A. Salazar* \\ * Departamento de Física Aplicada I, ETS Ingeniería, Universidad del País Vasco, UPV/EHU, Alda. Urquijo s/n, \\ 48013 Bilbao, Spain, arantza.mendioroz@ehu.eus \\ *Departamento de Matemática Aplicada EINA/UIMA, Universidad de Zaragoza, Campus Río Ebro, Edificio \\ Torres Quevedo, 50018 Zaragoza, Spain, celorrio@unizar.es \\ ***Instituto Politécnico Nacional, CICATA Legaria, Av. Legaria No. 694 Col. Irrigación, 11500 Mexico D.F., \\ Mexico
}

\begin{abstract}
Burst vibrothermography is used to characterize vertical cracks. We excite the sample with a short ultrasound burst of constant intensity and we register the evolution of the surface temperature during and after the burst. Among all this information, we characterize the crack using the thermogram obtained and the end of the burst and the evolution of the temperature (timing graph) at the center of the thermograms. We have developed a stabilized inversion algorithm to retrieve the area and depth of the crack. Inversions of experimental data obtained with samples containing calibrated heat sources confirm the validity of the method.
\end{abstract}

\section{Introduction}

The characterization of vertical kissing cracks in a fast way is a challenging task for non-destructive testing techniques. Lock-in vibrothermography has proven its ability to characterize vertical cracks but data taking is time consuming. In this work we propose burst vibrothermography, which is a much faster technique, to characterize vertical cracks. We start calculating the evolution of the surface temperature distribution produced by a vertical heat source. Then we propose to use the thermogram at the end of the burst and the timing graph at the center of the thermogram to characterize the crack. According to the ill-posed character of the inverse problem we have developed a stabilized inversion algorithm based on Tikhonov (TK), Total Variation (TV) and Lasso (L1) functionals to characterize the crack. We have checked the performance of the algorithm by inverting synthetic data with added white noise. Finally, inversions of experimental data obtained with samples containing calibrated heat sources confirm that the method is able to characterize vertical cracks in a fast way.

\section{Direct problem}

The evolution of the surface temperature distribution $(z=0)$ corresponding to a homogeneous vertical heat source of area $\Omega$, emitting a constant flux $Q$ during a time interval $\tau$ in a material or thermal conductivity $K$ and diffusivity $D$ is calculated by considering a semi-infinite sample and assuming adiabatic conditions at the surface [1]:

$$
\begin{array}{ll}
T_{h}\left(\vec{r}_{z=0}, t\right)=\iint_{\Omega} d \vec{r}_{0}^{3} \frac{Q}{2 \pi K\left|\vec{r}_{z=0}-\vec{r}_{0}\right|} \operatorname{ErfC}\left[\frac{\left|\vec{r}_{z=0}-\vec{r}_{0}\right|}{\sqrt{4 D t}}\right] & 0 \leq t \leq \tau \\
T_{c}\left(\vec{r}_{z=0}, t\right)=\iint_{\Omega} d \vec{r}_{0}^{3} \frac{Q}{2 \pi K\left|\vec{r}_{z=0}-\vec{r}_{0}\right|}\left\{\operatorname{ErfC}\left[\frac{\left|\vec{r}_{z=0}-\vec{r}_{0}\right|}{\sqrt{4 D t}}\right]-\operatorname{ErfC}\left[\frac{\left|\vec{r}_{z=0}-\vec{r}_{0}\right|}{\sqrt{4 D(t-\tau)}}\right]\right\} & t \geq \tau
\end{array}
$$

In a previous work [1] we analyzed the influence of the burst duration of the accuracy of the retrieved size and depth of rectangular vertical heat sources when assuming knowledge of the shape. We found that, in order to trust the results of the inversion for any burst duration, the number of data in the thermogram and in the timing graph should be of the same order. Following this idea, we have generated surface temperature data corresponding to different shapes and depths of the heat sources that we will use for the synthetic inversions presented in the next section.

\section{Inverse problem and inversions with synthetic data}

In the inverse problem we want to find the heat source distribution $Q(\vec{r})$ that minimizes the residual, i.e., the square differences between the data and the calculated temperatures. As we are dealing with an ill-posed inverse problem, the minimization process needs to be stabilized. Furthermore, since we want to use a quite limited amount of information (one thermogram and one timing graph) we have implemented the inversion algorithm by adding three regularization terms to the residual we want to minimize, one based on TK functional, one based on TV functional and another one based on Lasso (L1) functional, each of which multiplied by a regularization parameter $\left(\alpha_{T K}^{i}, \alpha_{T V}^{i}, \alpha_{L}^{i}\right)$, that we reduce in each iteration $i$ : 


$$
\alpha_{T K}^{i} T K_{0}(Q)+\alpha_{T V}^{i} T V(Q)+\alpha_{L}^{i} L_{1}(Q)+\left\|T_{\text {calc }}(Q)-T\right\|^{2}
$$

The initial value of all three regularization parameters is the same but they are reduced at different rates: $\alpha_{T K}^{i}=0.01^{i-1} \alpha, \alpha_{T V}^{i}=0.7^{i-1} \alpha, \alpha_{L_{1}}^{i}=0.6^{i-1} \alpha$. The Tikhonov term basically affects the first iteration and provides a first approximation of the heat source distribution, wider than the real one. Then, TV and L1 will act on this on successive iterations.

We have checked the performance of the inversion algorithm by inverting synthetic data with $5 \%$ added white noise corresponding to different shapes of the heat source, located at different depths and for different durations of the burst. As an example, in figure 1 we show a black and white representation of the heat source distribution for squares buried $200 \mu \mathrm{m}$ and $3 \mathrm{~mm}$ generated with bursts $\tau=100 \mathrm{~ms}, 500 \mathrm{~ms}, 1 \mathrm{~s}, 4 \mathrm{~s}$, and $8 \mathrm{~s}$.

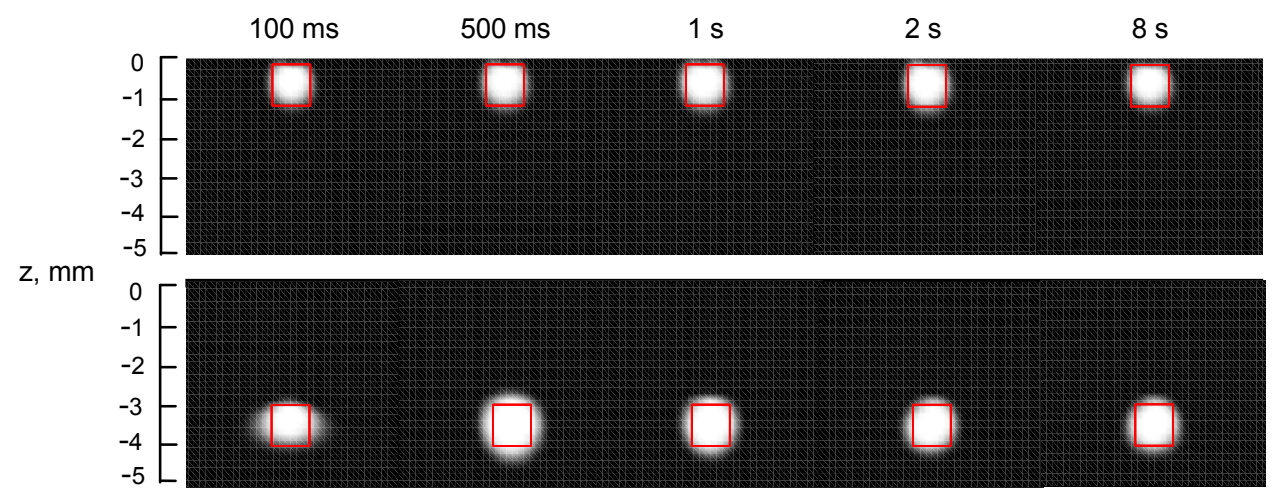

Fig. 1. Black and white representation of the heat source distribution obtained from synthetic data with $5 \%$ added white noise, corresponding to square heat sources buried $200 \mu \mathrm{m}$ (top), and $3 \mathrm{~mm}$ (bottom) for bursts of $0.1,0.5$, 1,2 and $8 \mathrm{~s}$. White is maximum value of the heat source distribution and black is absence of heat sources. Real contours are depicted in red.

As can be observed in figure 1, the reconstructions are very good for all burst durations and for depths down to $3 \mathrm{~mm}$. This confirms that the strategy of balancing space and time information is adequate to solve this problem.

\section{Experiments}

We have prepared samples containing calibrated heat sources by attaching two steel parts with a flat common surface and putting $38 \mu \mathrm{m}$ thick cooper slabs of known dimensions in-between. When we launch the ultrasounds there is friction between the cooper slabs and the steel surfaces. We have taken data for different depths of slabs with different geometries. In figure 2 we show as an example, the reconstructions we have obtained with a $1.5 \mathrm{~mm}$ side square buried $570 \mu \mathrm{m}$ below the surface, for different burst durations ranging between 0.5 and $6 \mathrm{~s}$. As can be observed, the quality of the reconstructions is very good. These results confirm that burst vibrothermography can be used to characterize vertical cracks in a fast way.

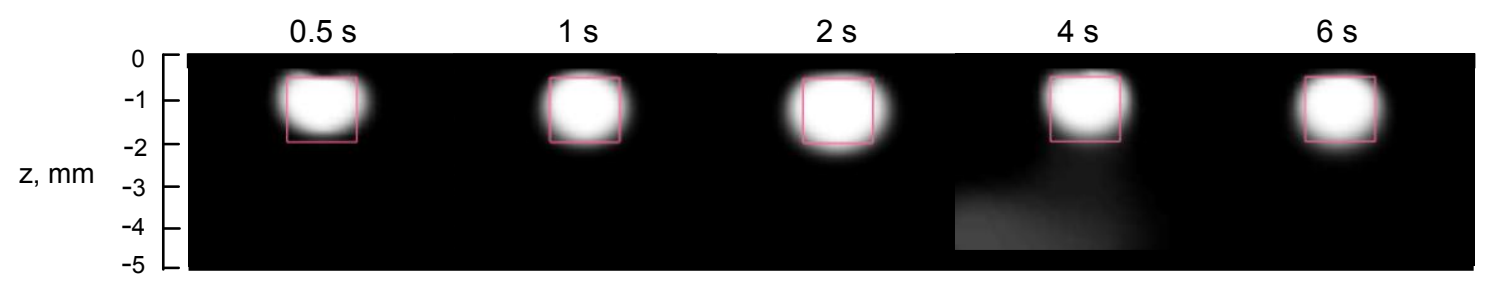

Fig. 2. Black and white representation of the normalized heat source distribution retrieved from experimental data obtained for different depths of a $1.5 \times 1.5 \mathrm{~mm}$ square Cu slab buried $570 \mu \mathrm{m}$. Real contours in red.

This work has been supported by the Ministerio de Ciencia e Innovación (MAT2011-23811), by Gobierno Vasco (IT61913), by UPV/EHU (UFI 11/55), and by CONACYT, beca mixta.

\section{REFERENCES}

[1] Mendioroz A., Celorrio R. and A. Salazar A., Characterization of rectangular vertical cracks using burst vibrothermography. Rev. Sci. Instr. - Vol. 86 (2015) pp. 064903-064903/9. 